\title{
Identifying possible inaccuracy in reported birth head circumference measurements among infants in the US Zika Pregnancy and Infant Registry
}

Nicole M. Roth (D YHW9@cdc.gov )

Division of Birth Defects and Infant Disorders, National Center on Birth Defects and Developmental Disabilities, Centers for Disease Control and Prevention, Atlanta, GA https://orcid.org/0000-0002-62795561

\section{Kate Russell Woodworth}

Division of Birth Defects and Infant Disorders, National Center on Birth Defects and Developmental Disabilities, Centers for Disease Control and Prevention, Atlanta, GA

\section{Shana Godfred-Cato}

Division of Birth Defects and Infant Disorders, National Center on Birth Defects and Developmental Disabilities, Centers for Disease Control and Prevention, Atlanta, GA

\section{Augustina M. Delaney}

Division of Birth Defects and Infant Disorders, National Center on Birth Defects and Developmental Disabilities, Centers for Disease Control and Prevention, Atlanta, GA; Eagle Global Scientific, LLC., Alpharetta, GA

\section{Samantha M. Olson}

Division of Birth Defects and Infant Disorders, National Center on Birth Defects and Developmental Disabilities, Centers for Disease Control and Prevention, Atlanta, GA; G2S Corporation, San Antonio, TX John F. Nahabedian III

Eagle Global Scientific, LLC., Alpharetta, GA

\section{Megan R. Reynolds}

Division of Birth Defects and Infant Disorders, National Center on Birth Defects and Developmental Disabilities, Centers for Disease Control and Prevention, Atlanta, GA

\section{Abbey M. Jones}

Division of Birth Defects and Infant Disorders, National Center on Birth Defects and Developmental Disabilities, Centers for Disease Control and Prevention, Atlanta, GA

\section{Varsha Neelam}

Division of Birth Defects and Infant Disorders, National Center on Birth Defects and Developmental Disabilities, Centers for Disease Control and Prevention, Atlanta, GA

\section{Miguel Valencia-Prado}

Puerto Rico Department of Health, San Juan, PR

\section{Camille Delgado-López}


Puerto Rico Department of Health, San Juan, PR

\section{Ellen H. Lee}

New York City Department of Health and Mental Hygiene, New York, NY

\section{Esther M. Ellis}

US Virgin Islands Department of Health, Christiansted, USVI

\section{Heather Lake-Burger}

Florida Department of Health, Tallahassee, FL

\section{Julius L. Tonzel}

Louisiana Department of Health, New Orleans, LA

\section{Cathleen A. Higgins}

Massachusetts Department of Public Health, Boston, MA

\section{Ronna L. Chan}

North Carolina Department of Health and Human Services, Raleigh, NC

\section{Van T. Tong}

Division of Birth Defects and Infant Disorders, National Center on Birth Defects and Developmental Disabilities, Centers for Disease Control and Prevention, Atlanta, GA

\section{Suzanne M. Gilboa}

Division of Birth Defects and Infant Disorders, National Center on Birth Defects and Developmental Disabilities, Centers for Disease Control and Prevention, Atlanta, GA

\section{Janet D. Cragan1}

Division of Birth Defects and Infant Disorders, National Center on Birth Defects and Developmental Disabilities, Centers for Disease Control and Prevention, Atlanta, GA

\section{Margaret A. Honein}

Division of Birth Defects and Infant Disorders, National Center on Birth Defects and Developmental Disabilities, Centers for Disease Control and Prevention, Atlanta, GA

\section{Cynthia A. Moore}

Division of Birth Defects and Infant Disorders, National Center on Birth Defects and Developmental Disabilities, Centers for Disease Control and Prevention, Atlanta, GA

\section{Research Article}

Keywords: Zika virus infection, pregnancy, congenital Zika syndrome, birth defects, population surveillance, microcephaly

Posted Date: December 22nd, 2021

DOl: https://doi.org/10.21203/rs.3.rs-1189991/v1

License: (c) (1) This work is licensed under a Creative Commons Attribution 4.0 International License. Read Full License 
Version of Record: A version of this preprint was published at Birth Defects Research on March 25th, 2022. See the published version at https://doi.org/10.1002/bdr2.1997. 


\section{Abstract}

The US Zika Pregnancy and Infant Registry (USZPIR) monitors infants born to mothers with confirmed or possible Zika virus (ZIKV) infection during pregnancy. The surveillance case definition for Zikaassociated birth defects includes microcephaly based on head circumference (HC). We assessed birth and follow-up data from infants with birth $\mathrm{HC}$ measurements $<3^{\text {rd }}$ percentile and birthweight $\geq 10^{\text {th }}$ percentile to determine possible misclassification of microcephaly.

We developed a schema informed by literature review and expert opinion to identify possible HC measurement inaccuracy using $\mathrm{HC}$ growth velocity and neuroimaging results. Two or more $\mathrm{HC}$ measurements between 2-12 months of age were required for assessment. Inaccuracy in birth HC measurement was suspected if growth velocity was $>3$ centimeters/month in the first three months or HC was consistently $>25^{\text {th }}$ percentile during follow-up. Normal neuroimaging was considered supportive of $\mathrm{HC}$ measurement inaccuracy.

Of 6,799 infants, 351 (5.2\%) had Zika-associated birth defects, of which 111 had birth HC measurements $<3^{\text {rd }}$ percentile and birthweight $\geq 10^{\text {th }}$ percentile. Of $84 / 111$ infants with sufficient follow-up, $38 / 84$ (45\%) were classified as having possible inaccuracy of birth $\mathrm{HC}$ measurement, $19 / 84(23 \%)$ had $\mathrm{HC} \geq 3^{\text {rd }}$ percentile on follow-up without meeting criteria for possible inaccuracy, and 27/84 (32\%) had continued $\mathrm{HC}<3^{\text {rd }}$ percentile. After excluding possible inaccuracies, the proportion of infants with Zika-associated birth defects including microcephaly decreased from $5.2 \%$ to $4.6 \%$.

About one-third of infants with Zika-associated birth defects had only microcephaly, but indications of possible measurement inaccuracy were common. Implementation of this schema in ZIKV infection during pregnancy studies can reduce misclassification of microcephaly.

\section{Full Text}

In November 2015, Brazil reported an association between Zika virus (ZIKV) infection during pregnancy and an increase in the number of infants born with microcephaly (Newton Sérgio De Carvalho, 2016). Very little was known about the potential harm ZIKV infection during pregnancy would have on pregnancy and infant outcomes. To better understand and define the spectrum of outcomes and provide guidance for pregnant women and their infants, the Centers for Disease Control and Prevention (CDC) collaborated with jurisdictional health department partners to establish the US Zika Pregnancy and Infant Registry (USZPIR) to prospectively follow pregnant women with laboratory evidence of confirmed or possible ZIKV infection and their infants born from December 1, 2015 through March 31, 2018 in the US states, District of Columbia, and US territories and freely associated states (Simeone, 2016; NCBDDD, 2019).

The USZPIR collects information from prenatal, birth hospitalization and delivery, and infant follow-up information up to ages 2-5 years from medical records. The surveillance case definition has been revised 
over time as our understanding of congenital ZIKV infection improved and now includes brain anomalies and/or microcephaly, eye abnormalities, and neurodevelopmental abnormalities (Rice, 2018; Olson, 2019). The surveillance case definition for microcephaly was defined as birth head circumference (HC) measurement less than the third percentile ( $<3$ rd percentile) for gestational age and sex based on INTERGROWTH-21 ${ }^{\text {ST }}$ standards (INTERGROWTH-21st, 2020) and has been in place since the inception (Honein, 2017).

The longitudinal surveillance structure of USZPIR allows information about infant health and developmental outcomes to accumulate over time, providing an opportunity for increased clinical detail, and presumably a more accurate clinical assessment. As additional infant follow-up visits were reported to USZPIR, the inherent challenges in conducting surveillance of microcephaly became more evident. Some infants with reported birth $\mathrm{HC}<3$ rd percentile had follow-up $\mathrm{HC}$ measurements that suggested the birth measurements were inaccurate. This analysis aimed to determine the possible inaccuracy of $\mathrm{HC}$ measurements among infants with birth $\mathrm{HC}<3$ rd percentile and birthweight $\geq 10$ th percentile who have additional follow-up data reported to USZPIR.

Clinical ZIKV subject matter experts developed a schema for reviewing microcephaly classification at birth among infants in USZPIR based on a review of the scientific literature and expert opinion (Box). Infants born small-for-gestational-age (SGA) (birthweight $<10$ th percentile by age and sex based on INTERGROWTH-21 ${ }^{\text {ST }}$ standards) were excluded from assessment due to differences in growth trajectory from infants born appropriate for gestational age (Karlberg, 1995). Birth $\mathrm{HC}<3$ rd percentile was evaluated for possible inaccuracy if two or more follow-up HC measurements were reported to USZPIR between two and twelve months of age with at least one measurement prior to six months of age; otherwise, infants were deemed to have insufficient data to assess. $\mathrm{HC}<3 \mathrm{rd}$ percentile was suspected to be inaccurate if either of two criteria was met: 1) greater than expected head growth velocity ( $>3 \mathrm{~cm} / \mathrm{month}$ ) between birth and three months of age (WHO, n.d.); or 2) all HCs above 25th percentile between two and twelve months of age. Normal neuroimaging reported from birth or infant follow-up was considered supportive evidence of possible birth $\mathrm{HC}$ measurement inaccuracy.

HCs at birth and throughout follow-up were reviewed and classified based on USZPIR data reported to $\mathrm{CDC}$ by December 2020. Eligible infants with birth $\mathrm{HC}<3$ rd percentile were categorized into one of four mutually exclusive categories based on follow-up HC measurements: 1) $\mathrm{HC}$ remains $<3$ rd percentile, 2) all follow-up HCs $\geq 3$ rd percentile and <10th percentile, 3) all follow-up HCs $\geq 10$ th percentile, and 4) suspected birth HC measurement inaccuracy. The proportion of infants in the USZPIR with Zikaassociated birth defects was calculated with and without infants suspected to have $\mathrm{HC}$ measurement inaccuracy at birth.

Among 6,799 liveborn infants in USZPIR with reported follow-up data, 252 infants had birth HC measurements $<3$ rd percentile (Figure 1). Of these, 141 (56\%) infants had $\mathrm{HC}<3$ rd percentile and a birthweight $<10$ th percentile (i.e., SGA). Among the remaining 111 infants with birthweight $\geq 10$ th percentile, 27 (24\%) infants had insufficient follow-up data to be assessed for birth HC inaccuracy. 
Among 84/111 infants with sufficient follow-up data (e.g., two or more reported $\mathrm{HC}$ measurements in follow-up), 27/84 (32\%) infants had $\mathrm{HC}$ measurements that remained <3rd percentile, 6/84 (7\%) infants had all follow-up HCs >3rd percentile and $<10$ th percentile, 13/84 (15\%) infants had all follow-up HCs $\geq 10$ th percentile, and $38 / 84$ (45\%) were suspected to have inaccuracy in the reported birth HC. Among infants with a suspected birth HC inaccuracy, none had reported brain abnormalities, and two had reported Zika-associated eye abnormalities. The proportion of infants with Zika-associated birth defects was $5.2 \%(351 / 6,799)$, and after accounting for the 36 infants with possible inaccuracy of birth HC measurement and no other reportable Zika-associated birth defect, the proportion of infants with Zikaassociated birth defects decreased to $4.6 \%(315 / 6,799)$.

Use of this schema for possible inaccuracy in reported birth $\mathrm{HC}$ measurement addresses misclassification and refines the prevalence estimate of Zika-associated birth defects in USZPIR. However, we were only able to apply this classification schema to a third $(33 \%, 84 / 252)$ of infants in USZPIR with a reported birth $\mathrm{HC}<3$ rd percentile, which demonstrates the challenges of defining and conducting surveillance of microcephaly.

Although microcephaly is often defined as birth $\mathrm{HC}<3$ rd percentile for gestational age and sex, a previous report reviewing the use of $\mathrm{HC}$ measurement alone for identification of microcephaly noted this criterion as practical for surveillance but found that when this cut-off-based definition was applied to populations, the observed prevalence contradicted the expected prevalence of microcephaly reported in the literature (Kalmin, 2019). Rounding of HC measurements to the whole centimeter, in addition to using only the number of completed weeks for gestational age, can introduce error and impact microcephaly prevalence both in routine surveillance prior to the Zika epidemic and even in situations during the Zika epidemic when staff are trained and insertion tapes are used for HC measurement (Harville, 2019; Harville, 2020). In the United States, birth defects surveillance programs use a variety of case ascertainment methods for identifying infants with microcephaly and typically use additional criteria beyond a $\mathrm{HC}$ measurement $<3 \mathrm{rd}$ percentile, such as documentation of a diagnosis of microcephaly in a medical record (Cragan, 2016).

The ZIKV outbreak showed the importance of conducting surveillance to describe the impact of congenital infections on the frequency of birth defects, including microcephaly. The USZPIR used a sensitive surveillance case definition for microcephaly enabling a greater detection of possible adverse outcomes associated with ZIKV infection during pregnancy, which was particularly important early in the outbreak when little was known about the effect of ZIKV infection during pregnancy. Surveillance case definitions for microcephaly that are sensitive and specific will help increase the likelihood of accurately describing these outcomes.

There are several limitations to this reclassification schema. First, almost a quarter of infants with birth $\mathrm{HC}<3$ rd percentile did not have enough follow-up information reported to reassess microcephaly classification at birth. Second, this reclassification schema fails to consider the timing of HC measurement after birth, which can influence the measurement. One study of 499 infants at birth found statistically significant differences between $\mathrm{HC}$ measurements taken at one and three days of life, as well 
as differences in HC dependent on the mode of delivery (Šimič Klaric', 2014). Finally, these criteria are not applicable to SGA infants since there are recognized differences in their growth trajectory. A considerable proportion of infants (56\%) in USZPIR meeting the case definition for birth $\mathrm{HC}<3$ rd percentile are proportionately SGA (head circumference $<3$ rd percentile and birth weight $<10$ th percentile). Future analyses will help investigate a possible association between prenatal exposure to ZIKV and SGA.

As additional information has become available, the USZPIR case definition for Zika-associated birth defects has adapted. The USZPIR surveillance case definition for microcephaly will continue to be defined as $\mathrm{HC}<3$ rd percentile for gestational age and sex reported during birth hospitalization. Infants in USZPIR that meet criteria for possible inaccuracy in birth $\mathrm{HC}$ will no longer be reported as having Zikaassociated birth defects if that is the only reportable abnormality. Infants without sufficient follow-up data to be assessed for inaccuracy will remain categorized as having a reported Zika-associated birth defect. Despite limitations and challenges, the USZPIR continues to provide surveillance data to inform pregnant women, families, healthcare providers, and public health professionals regarding the impact of ZIKV in pregnancy and the importance of recommended follow-up for timely referral to services.

Box. Schema for possible inaccuracy of head circumference $(\mathrm{HC})$ measurement among live born infants with birth $\mathrm{HC}<3$ rd percentile and birthweight $\geq 10$ th percentile reported to the US Zika Pregnancy and Infant Registry (USZPIR) ${ }^{\text {, } \neq}$

Infants must have two or more $\mathrm{HC}$ measurements reported between two and twelve months of age with the first measurement occurring prior to six months of age. A microcephaly $\mathrm{HC}$ measurement (HC $<3$ rd percentile) at birth is suspected to be inaccurate if either of the following criteria were met:

o Greater than expected head growth velocity ( $>3 \mathrm{~cm} / \mathrm{month}$ ) before three months of age $\S$

o Normal postnatal $\mathrm{HC}$ percentiles and consistently normal $\mathrm{HC}$ growth trajectory defined as postnatal $\mathrm{HC}$ consistently $>25$ th percentile during the first year of life

An infant with data that met either of the criteria is suspected to have an inaccurate $\mathrm{HC}$ reported at birth and no longer meets USZPIR surveillance criteria for microcephaly.

Normal neuroimaging was considered supportive evidence of birth HC measurement inaccuracy.

${ }^{\dagger}$ Infants with birthweight $<10$ th percentile, or small-for-gestational-age, typically have a growth trajectory different than infants with birthweight $\geq 10$ th percentile and thus were not evaluated using these criteria for possible $\mathrm{HC}$ measurement inaccuracy.

‡Percentiles by infant sex and gestational age based on INTERGROWTH-21st online percentile calculator.

SInfants with $>3$ centimeters per month of growth velocity would be in the 95th percentile for $\mathrm{HC}$ growth velocity, regardless of sex (WHO, n.d.).

\section{Declarations}


CDC Disclaimer: The findings and conclusions in this report are those of the authors and do not necessarily represent the official position of the Centers for Disease Control and Prevention.

The authors have no conflicts of interest to declare that are relevant to the content of this article.

\section{References}

1. Cragan, J. D., Isenburg, J. L., Parker, S. E., Alverson, C. J., Meyer, R. E., Stallings, E. B., ... National Birth Defects Prevention Network. (2016). Population-based microcephaly surveillance in the United States, 2009 to 2013: An analysis of potential sources of variation. Birth Defects Research Part $A$ Clinical and Molecular Teratology, 106(11), 972-982. doi: 10.1002/bdra.23587

2. De Carvalho, N. S., De Carvalho, B. F., Fugaça, C. A., Dóris, B., \& Biscaia, E. S. (2016). Zika virus infection during pregnancy and microcephaly occurrence: a review of literature and Brazilian data. The Brazilian Journal of Infectious Diseases, 20(3), 282-289. doi: 10.1016/j.bjid.2016.02.006

3. Harville, E. W., Buekens, P. M., Cafferata, M. L., Gilboa, S., \& Tong, V. (2019). Measurement error, microcephaly prevalence and implications for Zika: an analysis of Uruguay perinatal data. Archives of Disease in Childhood, 105(5), 428-432. doi: 10.1136/archdischild-2019-317678

4. Harville, E. W., Tong, V. T., Gilboa, S. M., Moore, C. A., Cafferata, M. L., Alger, J., ... Buekens P. (2020). Measurement of head circumference: implications for microcephaly surveillance in Zika-affected areas. Tropical Medicine and Infectious Disease, 6(1),5. doi: 10.3390/tropicalmed6010005.

5. Honein, M. A., Dawson, A. L., Petersen, E. E., Jones, A. M., Lee, E. H., Yazdy, M. M., ... Jamieson, D. J. (2017). Birth defects among fetuses and infants of US women with evidence of possible Zika virus infection during pregnancy. JAMA, 317(1), 59-68. doi: 10.1001/jama.2016.19006

6. INTERGROWTH-21st. Percentiles by infant sex and gestational age based on INTERGROWTH-21st online percentile calculator. Retrieved August 23, 2021 from http://intergrowth21.ndog.ox.ac.uk/

7. Kalmin, M. M., Gower, E. W., Stringer, E. M., Bowman, N. M., Rogawski McQuade, E. T., \& Westreich, D. (2019). Misclassification in defining and diagnosing microcephaly. Paediatric and Perinatal Epidemiology, 33(4), 286-290. doi:10.1111/ppe.12561

8. Karlberg, J. \& Albertsson-Wikland, K. (1995). Growth in full-term small-for-gestational-age infants: from birth to final height. Pediatric Research, 38(5), 733-739.

9. National Center on Birth Defects and Developmental Disabilities, Centers for Disease Control and Prevention. (2020, April 17). US Zika Pregnancy and Infant Registry. https://www.cdc.gov/pregnancy/zika/research/registry.html

10. Olson, S. M., Delaney, A., Jones, A. M., Carr, C. P., Liberman, R. F., Forestieri, N. E., ... Cragan, J. D. (2019). Updated baseline prevalence of birth defects potentially related to Zika virus infection. Birth Defects Research, 111, 938-940. doi:10.1002/bdr2.1546

11. Rice, M. E., Galang, R. R., Roth, N. M., Ellington, S. R., Moore, C. A., Valencia-Prado, M., ... Honein M.A. (2018). Vital Signs: Zika-associated birth defects and neurodevelopmental abnormalities possibly associated with congenital Zika virus infection - U.S. Territories and Freely Associated States, 2018. 
Morbidity and Mortality Weekly Report, 67, 858-867. doi:

http://dx.doi.org/10.15585/mmwr.mm6731e1

12. Simeone, R. M., Shapiro-Mendoza, C. K., Meaney-Delman, D., Petersen, E. E., Galang, R. R., Oduyebo, T., ... Zika and Pregnancy Working Group. (2016). Possible Zika virus infection among pregnant women - United States and Territories, May 2016. Morbidity and Mortality Weekly Report, 65, 514519. Doi: http://dx.doi.org/10.15585/mmwr.mm6520e1

13. Simič Klarić, A., Tomić Rajić, M., \& Tesari Crnković, H. (2014). Timing of head circumference measurement in newborns. Clinical Pediatrics, 53(5), 456-459. doi: 10.1177/0009922813510600

14. WHO, World Health Organization. (2021, February 22). Head circumference velocity. World Health Organization. https://www.who.int/tools/child-growth-standards/standards/head-circumferencevelocity

\section{Figures}




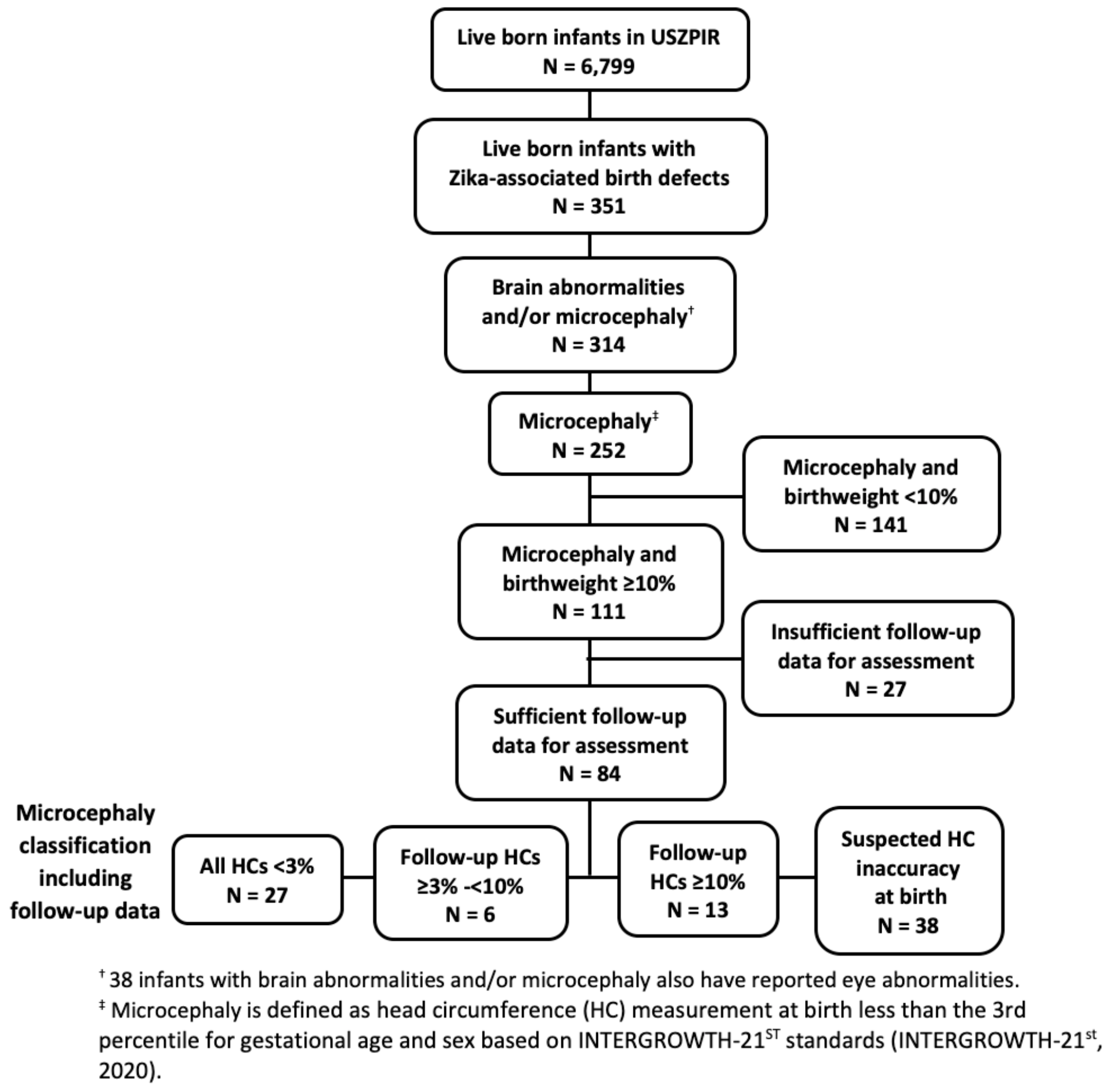

Figure 1

Live born infants in the US Zika Pregnancy and Infant Registry (USZPIR) with Zika-associated birth defects and classification of microcephaly using reported follow-up data 